Results 630 patients have outcomes recorded. We report on 370 who have completed treatment. $81 \%$ male, mean age 68 years (40-91). Patient's underwent mean 2.5 ablations (1-6) during protocol. 70\% baseline histology HGD, 27\% IMC \& 3\% LGD. Mean length baseline BE $5.6 \mathrm{~cm}(1-20)$. At 12 months CR-HGD was $87 \%$ patients, CR-D $82 \%$, \& CR-BE $64 \%$. $97 \%$ with no dysplasia at 12 months remain disease free at most recent follow up (median 18 months,range 2-68). Kaplan Meier statistics predict CR-D is durable at 5 years with $88 \%$ remaining disease free. Logistic regression demonstrate each extra 1 $\mathrm{cm}$ of $\mathrm{BE}$ reduces chances of attaining CR-D by $15.7 \%$ (OR 1.156, SE 0.048 , CI 1.07-1.26, $p=0.0003) \&$ for each extra RFA treatment likelihood of CR-D increases by $31.7 \%(\mathrm{OR}=0.683$, SE 0.95 , CI $0.52-0.89$, $\mathrm{p}=0.0006$ ). Progression to invasive cancer at 12 months is $2.7 \%$. Symptomatic strictures requiring dilatation occurred in $9 \%$ after treatment.

Conclusion End of protocol CR-D is encouraging at $83 \%$ \& successful eradication appears durable. Patients with shorter segment $\mathrm{BE}$ respond better \& multiple treatments are more likely to achieve CR-D. Our data represent real life outcomes of integrating novel endotherapy into demanding endoscopy service commitments

Disclosure of Interest None Declared

\section{OC-052 COMBINED EMR AND RADIO FREQUENCY ABLATION LEADS TO HIGH BARRETT'S ERADICATION RATES FOLLOWING STRUCTURED TRAINING PROGRAMME}

doi:10.1136/gutjnl-2013-304907.051

'J Faulkner, 'R Ley Greaves, 1."J Hoare. 'Gastroenterology, Imperial College London, London, UK

Introduction EMR and Radio Frequency Ablation (RFA) have recently been combined to treat dysplastic Barrett's oesophagus (1). These are complex techniques and require a high level of endoscopic skill and published reports show a range of success. The Academic Medical Centre (AMC) in Amsterdam is a high volume tertiary centre for these procedures and has established expertise in providing structured teaching (2). After attending a structured teaching programme at the AMC a service was established at a London teaching hospital to treat patients with dysplastic Barrett's oesophagus. We wanted to know if high quality results could be reproduced in this setting.

Methods We retrospectively analysed all cases of dysplastic Barrett's referred for treatment at our centre since the introduction of RFA (Barryx), following structured training at the AMC. Decision for endoscopic therapy was made at a multidisciplinary meeting involving surgeons, radiologists, oncologists and gastroenterologists. Published protocols for treatment with EMR/RFA were closely followed (1), although argon plasma coagulation was used to remove residual islands less than $5 \mathrm{~mm}$ in the interests of cost, rather than RFA. All procedures were carried out by one of two senior endoscopists.

Results Over 30 months 33 patients were referred for endoscopic therapy. Following initial EMR of visible lesions 3were found to have cancer extending beyond the first $1 / 3$ of the sub-mucosa and were offered alternative therapy. 24have finished therapy and 1 is lost to follow up. Mean age was 70 years (53-89) and mean Barrett's length $5.4 \mathrm{~cm}(<1-10 \mathrm{~cm})$. Therapy was applied as follows: 2 patients had only EMR, 4 only RFA, 1 EMR + APC, 6 EMR + RFA, 5 RFA +APC, 6 EMR + RFA + APC. 24/24 have had eradication of high grade dysplasia or intra-mucosal cancer (100\%). 21/24(87.5\%) have had complete eradication of Barrett's by endoscopic and histological criteria. Mean follow up is 9.8 months(1.5-25). There were no perforations. 3 strictures were treated endoscopically.

Conclusion Following a comprehensive structured teaching programme in the treatment of dysplastic Barrett's with combined RFA and EMR, results comparable to published studies are achievable in lower volume centres treating approximately only one new patient per month.

Disclosure of Interest None Declared

\section{REFERENCES}

1. Radiofrequency ablation for total Barrett's eradication: a description of the endoscopic technique, itsclinical results and future prospects. Pouw RE et al. Endoscopy. 2008 Dec; 40(12):1033-402.

2. Learning to perform endoscopic resection of esophageal neoplasia is associated with significant complications evenwithin a structured training programme. Van Vilsteren FG et al. Endoscopy. 2012 Jan; 44(1):4-12.

\section{Liver symposium: impact of clinical research in hepatology}

\section{OC-053 CURCUMIN, ANTI-OXIDANT, AND PIOGLITAZONE THERAPY WITH INCLUSION OF VITAMIN E IN NON ALCOHOLIC FATTY LIVER DISEASE-A RANDOMIZED OPEN LABEL PLACEBO CONTROLLED CLINICAL PROSPECTIVE TRIAL (CAPTIVE)}

doi:10.1136/gutjnl-2013-304907.052

1,2,"P Basu, ${ }^{3} \mathrm{~N}$ J Shah, ${ }^{2} \mathrm{~S}$ Farhat, ${ }^{1} \mathrm{R}$ Siriki, ${ }^{1} \mathrm{~K}$ Mittimanj, ${ }^{1} \mathrm{~S}$ Atluri, ${ }^{1} \mathrm{M}$ Rahaman. ${ }^{1}$ Forest Hills Hospital, Hofstra North Shore-LIJ School of Medicine; ${ }^{2}$ Columbia School of Physi-

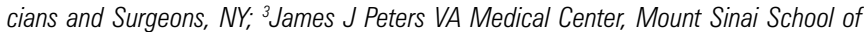
Medicine, New York, New York, United States

Introduction NAFLD is a global clinical challenge which progresses to cirrhosis and liver cancer. Defective transport of free fatty acids and mitochondrial dysfunction lead to explosion of a series of free radicals, apoptosis, up regulated cytokines and fibrogenesis ultimately causing cirrhosis and cancer. Curcumin is a pan-antioxidant with anti-inflammatory, anti-apoptotic, anti-microbial, and antifibrogenic properties. This study evaluates the role of curcumin in NAFLD to progression of NASH

Methods Eighty patients $(\mathrm{n}=80)$ with mean BMI $29 \%$, NAFLD score 0.66, NASH fibrotic score 0.33, HOMA IR 3.8, ALT 58, LDLc 143, HDLc 29, Triglyceride 186 and Adipokines (leptin, Adiponectin, Retinal Binding Proteins) were divided into Group A- $(n=20)$ pioglitazone $15 \mathrm{mg}$, Group B- $(\mathrm{n}=20)$ vitamin E, Group C- $(\mathrm{n}=20)$ curcumin (all the three above groups received placebo), and Group $D(n=20)$ vitamin $E$ plus curcumin. Pre and post values (Triglycerides, LDLc, HDLc, ALT, HOMA-IR, TNF-alfa, Leptin, Adiponectin, Retinol Binding Protein, HBA1c, Serum necro-inflammatory NAFLD and NASH fibrotic score were analysed at 3, 6, and 12 months. Diet and exercise were left unchanged. Daily alcohol content was less than 30 grammes

Results Group A-Minimal changes on ALT, HbA1c, HOMA, lipids, no changes in TNF-alfa, adipokines, lipid profile and necro-inflammatory score and/or NASH fibrosis score. Group B and Group C had modest changes in ALT, lipid profile, HbA1c and HOMA; while no changes in adipokines, necro-inflammatory score and fibrotic score. Group D had significant changes in all scores particularly the adipokines and small improvements in fibrotic score. All patients tolerated the medications well

Conclusion This study postulates the effects of Curcumin plus vitamin $\mathrm{E}$ in NAFLD may prevent NASH with a modest anti-fibrotic effects and necroinflammatory score; with impressive changes in adipokines levels. Additive effects of Curcumin with vitamin E has significant effects on Serum lipids and insulin sensitivity. Unavailability of Pre and post liver biopsy was the limitation A large control trial needs to validate.

Disclosure of Interest None Declared

\section{OC-054 HEPATIC EXPRESSION OF CCL25 MEDIATES RECRUITMENT OF PLASMACYTOID DENDRITIC CELLS TO LIMIT LIVER INJURY}

doi:10.1136/gutjnl-2013-304907.053

${ }^{1} \mathrm{D}$ Reid, ${ }^{2} \mathrm{~V}$ Lai, ${ }^{3} \mathrm{C}$ Weston, ${ }^{1} \mathrm{~T}$ Vo, ${ }^{4} \mathrm{M}$ Peters, ${ }^{3} \mathrm{D}$ Adams, 1, B B Eksteen. ${ }^{1}$ Snyder Institute for Chronic Diseases, University of Calgary, Calgary, Canada; ${ }^{2}$ Asian Centre for Liver Disease \& Transplantation, Singapore, Singapore; ${ }^{3}$ Centre for Liver Research, NIHR Biomedical Research Unit, University of Birmingham, Birmingham, UK; ${ }^{4}$ University of California, San Francisco, United States 
Introduction Primary Sclerosing Cholangitis (PSC) is an incurable chronic immune mediated biliary disease that occurs in individuals with IBD. We have previously reported aberrant expression of the gut chemokine, CCL25 in the human PSC liver and the recruitment of CCR9 expressing gut derived T cells. In order to gain further insights into the consequences of aberrant CCL25 expression in the liver in PSC, we induced CCL25 in the murine liver and assessed biliary inflammation in-vivo.

Methods To clarify the functional role of CCL25 expression in the liver, we generated a murine liver specific knock-in of CCL25 expression and tested the effects on immune mediated cholangitis using the Ova-Bil model of antigen driven biliary injury. Immune cell phenotyping and isolation were performed using flow cytometry. Liver injury was assessed by ALT measurements and histopathology. pDC function was assessed ex-vivo in co-culture with naive transgenic TCR T cells

Results Ova-Bil x CCL25KI mice developed significantly less liver injury than wt Ova-Bil controls. Flow cytometry revealed increased numbers of CCR9 ${ }^{+}$pDCA- $1^{+}$plasmacytoid dendritic cells ( $\mathrm{pDC}$ ) in the Ova-Bil x CCL25KI livers. CCR9-/ x Ova-Bil mice developed significantly worse liver injury compared to wt Ova-Bil controls and severely lacked $\mathrm{pDCs}$ in the liver. Adoptive transfer of $w t$ pDCs to CCR9 $\%$ x Ova-Bil mice rescued the phenotype and reduced the degree of liver injury comparable to wt Ova-Bil controls. In vitro studies demonstrated the ability of liver-derived $\mathrm{pDC}$ so induce regulatory $\mathrm{T}$ cells in a retinoic acid dependent manner as a possible mechanism by which CCR9 ${ }^{+}$pDCs are able to control liver injury.

Conclusion Aberrant expression of CCL25 in the liver enhances recruitment of $\mathrm{CCR}^{+}{ }^{+} \mathrm{pDCs}$ and appears to be an attempt to limit the extent of hepatic inflammation in PSC. Regulatory effects of $\mathrm{CCR}^{+}$pDCs appears to be at least in part mediated through the expansion of hepatic regulatory $T$ cells.

Disclosure of Interest None Declared

\section{REFERENCE}

1. Eksteen B, Grant AJ, Miles A, et al. Hepatic endothelial CCL25 mediates the recruitment of CCR9 + gut-homing lymphocytes to the liver in primary sclerosing cholangitis. J.Exp.Med. 2004; 200:1511-1517.

\section{Endoscopy symposium: endoscopy in the management of obesity}

\section{OC-055 A NOVEL TECHNIQUE FOR FULL THICKNESS LAPAROENDOSCOPIC EXCISION OF COLONIC LESIONS: AN EXPERIMENTAL PILOT STUDY}

doi:10.1136/gutjnl-2013-304907.054

\begin{abstract}
$1,2,{ }^{*} \mathrm{~A}$ Brigic, ${ }^{3} \mathrm{~A}$ Southgate, ${ }^{3} \mathrm{P}$ D Sibbons, ${ }^{1} \mathrm{C}$ Fraser, ${ }^{2} \mathrm{~S} \mathrm{~K}$ Clark, ${ }^{2} \mathrm{R} \mathrm{H}$ Kennedy. ${ }^{1}$ Wolfson Endoscopy Unit; ${ }^{2}$ Department of Surgery, St. Mark's Hospital and Academic Institute; ${ }^{3}$ Bioscience, Northwick Park Institute for Medical Research, London, UK
\end{abstract}

Introduction Introduction of a National Bowel Cancer Screening Program in England has resulted in an increasing number of patients diagnosed with endoscopically irresectable colonic polyps. A significant proportion of these patients is referred for hemicolectomy and is subject to a significant risk of morbidity and mortality. Therefore, a less invasive treatment option is required and to address this, we modified a previously reported full thickness laparo-endoscopic excision (FLEX) technique.

Methods Surgery was performed in five $70-\mathrm{kg}$ pigs. A simulated colonic polyp was created by endoscopic injection of Spot ${ }^{\circledR}$ and the clearance margin delineated by circumferential placement of mucosal argon plasma coagulator (APC) marks. Full thickness eversion of the colonic wall, including the lesion, was achieved by endoscopic placement of prototype BraceBars (BBs). The everted section was excised using a linear laparoscopic stapler placed below the BBs. The first pig was terminated immediately and others were sacrificed 8 days after surgery.

Results The median procedure duration, defined from placement of mucosal APC marks to specimen excision, was 26 min (range 20-31 min). All excised specimens contained three pairs of BBs, included the APC marks and had a median diameter of $5.1 \mathrm{~cm}$ (range $4.5-6.3 \mathrm{~cm}$ ). Postoperative recovery in survival animals was uneventful. Post-mortem evaluation demonstrated well-healed resection sites with no evidence of intra-abdominal infection or inadvertent organ damage. Endoscopic evaluation of anastomoses at post-mortem demonstrated a widely patent lumen without evidence of stenosis at excision sites. Histological examination of the anastomoses showed primary closure by mucosal abbutal and regeneration, with repair and restoration of submucosal continuity. Conclusion This proof-of-concept study has demonstrated the feasibility and safety of a novel full thickness colonic excision technique that is now ready for translation as an alternative to hemicolectomy. The excision size will accommodate most colonic polyps that currently come to surgery. Accurate placement of endoscopic $\mathrm{BBs}$ ensures complete excision, reducing the risk of residual disease and recurrence, while laparoscopic overview avoids collateral damage. The ability to preserve mesenteric vasculature and colonic length is likely to result in less morbidity and mortality, better functional outcomes and the approach should reduce treatment costs.

Disclosure of Interest None Declared

\section{OC-056 LONG-TERM TRENDS IN COMORBIDITY AND RISK SCORES AND THEIR INFLUENCE ON OUTCOMES OF UPPER GASTROINTESTINAL BLEEDING}

doi:10.1136/gutjnl-2013-304907.055

1." A S Taha, 'E Saffouri, ${ }^{2} \mathrm{C}$ McCloskey, ${ }^{2} \mathrm{~T}$ Craigen, ${ }^{3} \mathrm{~W}$ J Angerson. ${ }^{1}$ Medicine, University of Glasgow, Glasgow; ${ }^{2}$ Medicine, University Hospital Crosshouse, Kilmarnock; ${ }^{3}$ Surgery, University of Glasgow, Glasgow, UK

Introduction The prevention of upper gastrointestinal bleeding (UGIB) can be facilitated by understanding the changes in environmental and socio-pathological factors; these might not become obvious in short-term studies. We, therefore, aimed to study the changes in comorbidity and risk scores and their influence on the outcomes of UGIB over a 14-year period.

Methods We analysed the clinical characteristics of all patients presenting with UGIB to a single institution, 1996-2010. The Charlson's comorbidity and the complete Rockall scores were analysed, together with patients' drug use and 30-day mortality. Trends with time were assessed using logistic regression analysis with year of presentation as a continuous predictor variable. Regression coefficients were expressed as odds ratios (OR), representing the relative change in odds of death or other binary dependent variables over a time interval of one year.

Results A total of 2669 patients were included. The Charlson score increased significantly with time $(\mathrm{P}<0.001)$, the odds of a high $(3+)$ score increasing at a relative rate of $4.4 \%$ a year $(\mathrm{OR}=1.044,95 \% \mathrm{CI}$ $1.022-1.065)$. No significant trend with time was noted for age $(p=0.09)$, haemoglobin level $(P=0.47)$ or Rockall score $(P=0.94)$. The overall 30 -day mortality was $4.9 \%$ and this showed no relationship with time $(\mathrm{P}=0.28)$. However, when adjusted for the increasing comorbidity, the odds of death within 30 days decreased significantly at a relative rate of $4.5 \%$ per year $[\mathrm{OR}=0.955(0.914-0.997)$; $\mathrm{P}=0.038]$. Trends in the prevalence of taking potentially damaging and protective drugs are shown in Table-1, below. The rise in use of aspirin, other anti-thrombotic drugs and SSRIs [with pro-UGIB activity] was paralleled by a rise in the use of PPIs [protective activity] and beta-blockers, ACE inhibitors, and statins [being able to affect mortality. 